\title{
Two-color photoionization in xuv free-electron and visible laser fields
}

\author{
M. Meyer, ${ }^{1}$ D. Cubaynes, ${ }^{1}$ P. O'Keeffe, ${ }^{1}$ H. Luna, ${ }^{2}$ P. Yeates, ${ }^{2}$ E. T. Kennedy, ${ }^{2}$ J. T. Costello, ${ }^{2}$ P. Orr, ${ }^{3}$ R. Taïeb, ${ }^{4}$ \\ A. Maquet, ${ }_{1}^{4}$ S. Düsterer, ${ }^{5}$ P. Radcliffe,${ }^{5}$ H. Redlin,${ }^{5}$ A. Azima, ${ }^{5}$ E. Plönjese, ${ }^{5}$ and J. Feldhaus ${ }^{5}$ \\ ${ }^{1}$ LIXAM/CNRS, Centre Universitaire Paris-Sud, F-91405 Orsay Cedex, France \\ ${ }^{2}$ National Center for Plasma Science and Technology and School of Physical Sciences, Dublin City University, Dublin, Ireland \\ ${ }^{3}$ International Research Centre for Experimental Physics (IRCEP), Queen's University Belfast, BT7 INN, United Kingdom \\ ${ }^{4}$ Laboratoire de Chimie Physique-Matière et Rayonnement, UMR 7614, Université Pierre et Marie Curie, 11 Rue Pierre et Marie Curie, \\ 75231 Paris Cedex 05, France \\ ${ }^{5}$ HASYLAB at DESY, Notkestrasse 85, D-22603, Hamburg, Germany \\ (Received 27 March 2006; published 14 July 2006)
}

\begin{abstract}
Two-photon ionization of atomic helium has been measured by combining femtosecond extreme-ultraviolet pulses from the free-electron laser in Hamburg (FLASH at DESY) with intense light pulses from a synchronized neodymium-doped yttrium lithium fluoride laser. Sidebands appear in the photoelectron spectra when the two laser pulses overlap in both space and time. Their intensity exhibits a characteristic dependence on the relative time delay between the ionizing and the dressing pulses and provides an inherent time marker for time-resolved pump-probe experiments. The measurements of the sidebands are in good agreement with theoretical predictions and allow for a direct analysis of two-photon ionization, free from processes related to interference between multiple quantum paths.
\end{abstract}

DOI: 10.1103/PhysRevA.74.011401

PACS number(s): $32.80 . \mathrm{Rm}, 32.80 . \mathrm{Fb} ., 42.50 . \mathrm{Hz}$

The achievement of short-wavelength free-electron-laser (FEL) action at DESY in the year 2000 [1], based on the process of self-amplified spontaneous emission (SASE), represented a synergistic tour de force as optical and accelerator technologies were combined to produce ultrashort laser pulses at high fundamental photon energies with high peak and average power. The first experiments on rare-gas clusters revealed new insights into intense laser-matter interactions [2]. In contrast to visible and infrared laser-matter interactions, where valence electrons are the primary participants, the fundamental FEL photon energy lies far above the ionization potential of all stable matter. Under these conditions, inner-shell electrons can be excited into resonant and nonresonant continuum states (e.g., $[3,4]$ ) and will be the predominant mediators of the underlying photoprocesses (linear and nonlinear).

Starting in mid-2005 the free-electron laser in Hamburg (FLASH) covers a much larger wavelength range compared to the first lasing in 2000. In view of its unprecedented characteristics [5], an associated time-synchronized optical laser facility opens up new and particularly exciting research opportunities allowing the investigation of fundamental photoionization processes in intense bichromatic laser fields where one field can directly ionize valence and/or inner-shell electrons in a single step. The dynamics of various processes can be investigated, including ultrafast electronic relaxation of autoionization states [6], coupling between two autoionization states $[7,8]$, wave-packet formation of high-lying Rydberg states [9], fast dissociation of molecules upon inner- and outer-shell photoexcitation, etc. Such a pump-probe setup has recently been implemented at the FEL facility at DESY, which provides either femtosecond or picosecond infraredvisible pulses, synchronized with a rms jitter of less than 1 ps to the femtosecond xuv pulses from the FEL [10]. Here, we

present experimental results obtained with this system, combined with a corresponding theoretical analysis, on the photoionization of the most prototypical of atoms for such studies-helium - in the presence of a strong optical field.

Although studies of two-color (xuv and visible-infrared) photoionization of atoms in weak fields are well established (see, e.g., [11,12]), it is only recently that experiments have been performed, in which electrons ejected by the xuv field are born into an intense optical laser (or "dressing") field. The photoionization dynamics were studied in detail both experimentally and theoretically [13-17]. In these cases, continuum electrons can exchange one or more dressing laser photons, and sidebands are observed in the photoelectron spectrum, a phenomenon similar to the so-called abovethreshold ionization (ATI) process. It has been shown, notably in experiments performed with high-order harmonics, that the intensities of the resulting sidebands in the photoelectron spectra exhibit a strong dependence on the time separation and shapes of the xuv and visible-infrared pulses.

In sharp contrast to these studies, the xuv radiation from the FEL is essentially monochromatic and the xuv pulse energy is such that single-shot data are attainable. Several additional points are worthy of note. (i) The two-photon processes producing the sidebands in the photoelectron spectra are free from interference effects and partial two-photonionization cross sections into the $\mathrm{He}^{+}(1 s)+\epsilon s, \epsilon d$ continua are directly studied. (ii) The sideband intensity provides a clear indication of the temporal overlap between the FEL and the optical laser beams as well as their temporal stability. (iii) The experiment and results constitute the opening up of a pathway toward even more challenging experimental and theoretical investigations with ultrafast, intense polychromatic fields.

In order to observe the two-photon ionization signals we employed single-shot (FEL) photoelectron spectroscopy using a magnetic bottle electron spectrometer. This setup was used recently for one-photon experiments [18]. The electrons created in the acceptance volume of the analyzer after inter- 


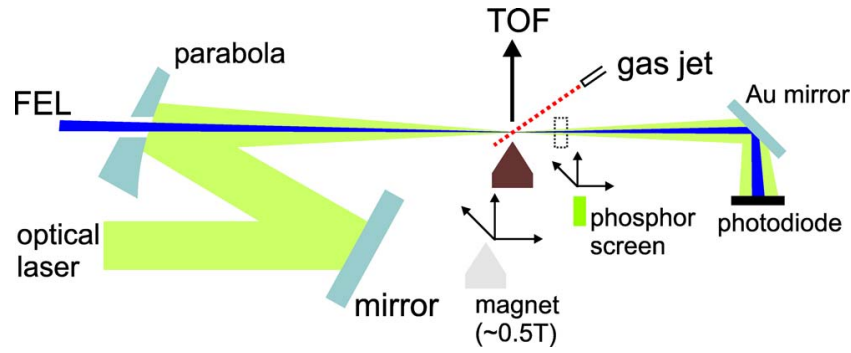

FIG. 1. (Color online) Experimental setup for the pump-probe experiments using the combination of the FEL with an optical laser.

action of the xuv light with an effusive atomic gas jet are directed toward a time-of-flight (TOF) tube by a strong magnetic field $(0.5 \mathrm{~T})$ provided by a conical-shaped permanent magnet (Fig. 1). They are detected by a microchannel plate detector used in current mode. Full angular acceptance is achieved by this configuration, allowing an analysis of the photoionization process on a shot-to-shot basis.

The FEL was operated at $32.2 \mathrm{~nm}$ and a repetition rate of $2 \mathrm{~Hz}$ for the pulse trains consisting of up to 14 individual pulses (1 $\mu$ s spacing), which had a temporal width of less than $50 \mathrm{fs}$ and energies of up to $40 \mu \mathrm{J}$ [5]. The optical beam was introduced in a collinear geometry into the experimental chamber (Fig. 1). Beam diameters of both fields at the focus position were about $50 \mu \mathrm{m}$. The corresponding xuv field intensity was about $7 \times 10^{11} \mathrm{~W} \mathrm{~cm}^{-2}$. For the two-color experiments a frequency-doubled neodymium-doped yttrium lithium fluoride (Nd:YLF) laser ( $250 \mu \mathrm{J}$ in 12 ps at $523 \mathrm{~nm}$ ) was used at this time as it produced a sufficiently intense field to induce a measurable ATI signal, while relaxing the constraint on temporal synchronization between both pulses. A phosphor screen was used for optimizing visually the spatial overlap of the FEL and the optical laser. The time delay between the xuv and the optical laser pulses was varied by passing the green beam through a variable optical delay. In order to measure this temporal delay at the actual interaction region in the experimental chamber we used a fast photodiode to yield a coarse time delay measurement with an accuracy of $50 \mathrm{ps}$ and the variation in the sideband signal to subsequently obtain a fine subpicosecond timing measurement. The time jitter between the FEL and optical laser was monitored by combining the incoherent radiation from the accelerator together with $0.1 \%$ of the Nd:YLF radiation onto the slit of a fast streak camera. In this way any pulse-to-pulse jitter and long-term drift could be measured with better than $1 \mathrm{ps}$ (rms) resolution.

In Fig. 2, a typical one-photon-ionization spectrum of atomic He recorded with the FEL beam is shown. The photoelectron spectrum is dominated by a strong emission line at a kinetic energy of about $14 \mathrm{eV}$ resulting from direct photoionization in the $\mathrm{He} 1 s$ shell by the FEL radiation at $\hbar \omega(\mathrm{FEL})=38.5 \mathrm{eV}$. The spectrum is similar to that which would be typically obtained using a synchrotron radiation source, apart from some intriguing weak features observed at higher kinetic energies (given in the inset). These latter structures could be due either to the photoionization of $\mathrm{He} 1 s^{2}$ by the second and third harmonics of the FEL fundamental frequency [18] or to nonlinear two- or three-photon absorption

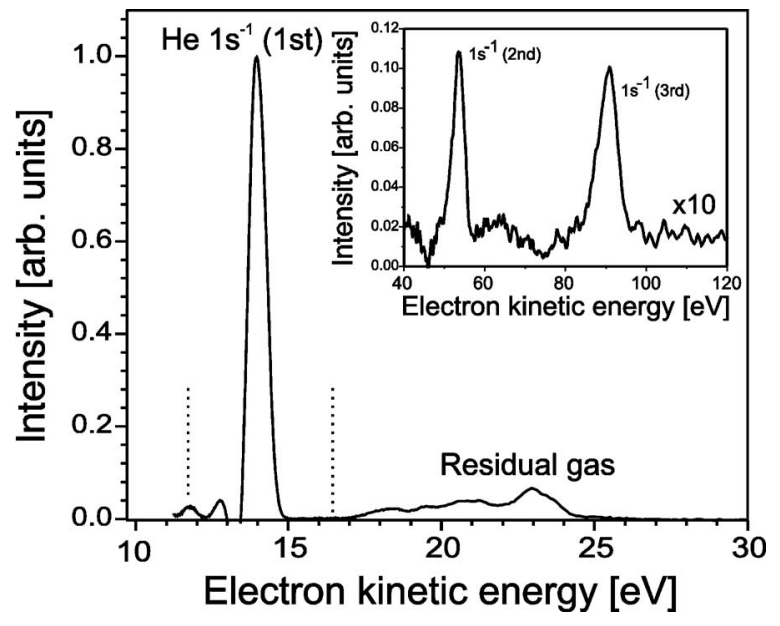

FIG. 2. Photoelectron spectrum for the one-photon ionization of He atoms by the xuv radiation from the FEL. The energy positions where sidebands created by the additional dressing field would be observed are indicated by the dotted lines. The inset shows the high-kinetic-energy part of the spectrum.

from the fundamental frequency. These two different processes would produce photoelectron lines at the same kinetic energies and cannot be distinguished in our experiment. However, given the present characteristics of the FEL $\left(I_{\mathrm{fel}} \approx 7 \times 10^{11} \mathrm{~W} \mathrm{~cm}^{-2}\right)$, we theoretically predict that the two-photon ionization signal for He would amount to only $10^{-6}$ of the single-photon absorption (see Fig. 4). Therefore we conclude that the peaks in the inset of Fig. 2 are due solely to single-photon ionization by the FEL's harmonics and lead to an estimate of about $0.5 \%$ for the relative intensities of the second- and third-order harmonics compared to the fundamental, as deduced using the known cross sections for one-photon ionization of He as a function of energy.

The transition probability for two-color ATI processes combining xuv and visible or infrared photons is, however, much higher than for multi-xuv photon processes, as freefree transitions scale with the inverse fourth power of the frequency [19]. A typical set of such two-color, two-photon photoelectron spectra, showing the region close to the $\mathrm{He}$ $1 s^{-1}$ main line, is displayed in Fig. 3 for different values of the temporal delay between the xuv and the visible light pulses, and shows clearly the growth and decay of the sidebands as the interpulse temporal delay is varied over the duration of the optical laser pulse.

We stress that sidebands on both sides of the main photoelectron peak are produced. However, since the low-energy sideband overlaps with the signal resulting from the ionization of residual species, in Fig. 3 only the sideband on the high-kinetic-energy side is shown. An average over 64 single-shot spectra is displayed in order to bring out more clearly the sideband. The observed changes in the shape of the sideband peak are caused by the relatively poor statistics and by the strong variation of the spectral profile of FEL pulses [18]. The average intensity of the sideband is of the order of $2 \%$ of the main $\mathrm{He} 1 s^{-1}$ photoline with an uncertainty of about $\pm 1 \%$. This small value is due to the optical laser pulses $(12 \mathrm{ps}, 250 \mu \mathrm{J})$ producing a field of only about $2 \times 10^{11} \mathrm{~W} \mathrm{~cm}^{-2}$, which is close to the lower threshold for 


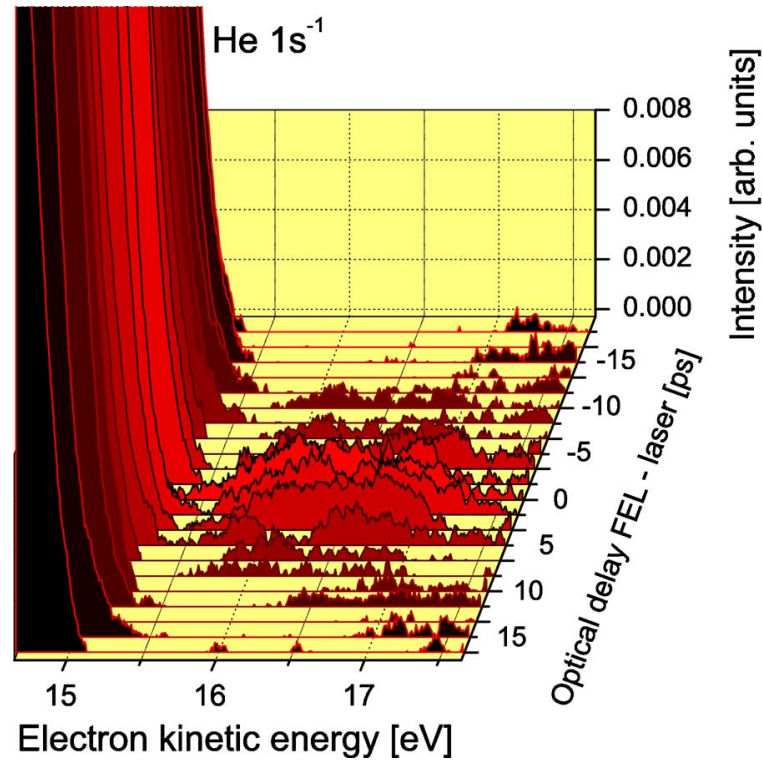

FIG. 3. (Color online) Part of the photoelectron spectrum in the region of the He $1 s^{-1}$ main line and the high-energy sideband. Different spectra are given as a function of the time delay between the pulses of the FEL and the optical (12 ps, $523 \mathrm{~nm})$ laser.

creating sideband structures in a photoelectron spectrum.

The experimental data can be interpreted in terms of a theoretical analysis performed by numerically solving the time-dependent Schrödinger equation (TDSE) for a singleactive-electron, three-dimensional model of $\mathrm{He}$ [20]. The results of this simulation, corresponding to the present experimental conditions, are shown in Fig. 4, where we have displayed the photoelectron spectrum calculated from the spectral analysis of the wave function at the end of the pulses. Since the duration of the optical laser is much longer than that of the xuv pulse, the ratio between the sideband and the main peak does not depend on the temporal shape of the FEL and has not been taken into account in the TDSE. The

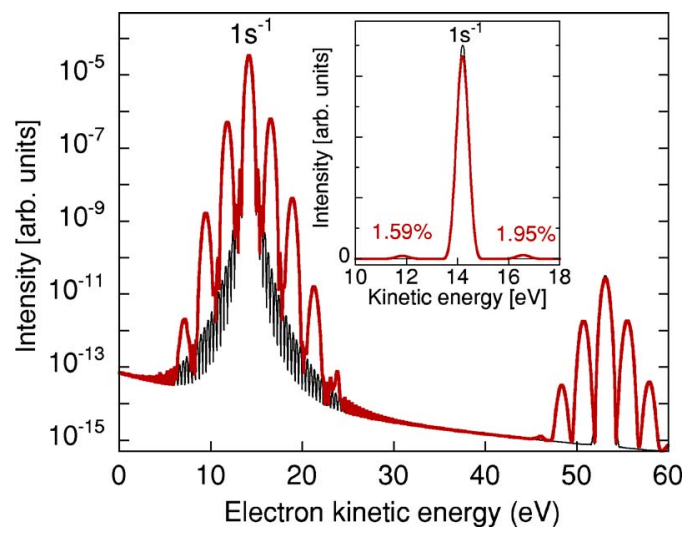

FIG. 4. (Color online) Theoretical photoelectron spectrum of He obtained from TDSE calculations for the present characteristics of the FEL and optical laser. The interaction with the FEL radiation leads to one-photon and two-photon ionization of the He $1 s$ shell (thin black line); the optical laser field creates the additional sideband structures (bold red line). The inset shows the He $1 s^{-1}$ photoline and the first sidebands on a linear intensity scale. log-scale figure shows a dominant peak corresponding to the absorption of a single photon from the xuv field. It is accompanied by a set of symmetrical sidebands (separated by the optical photon energy) with intensities that decrease by at least two orders of magnitude when going from one sideband to the next. As shown in the inset, only the first sidebands are visible on a linear scale relevant for comparison with the experiment. The relative magnitude of the right-hand sideband, as compared to the main peak, is found to be $1.95 \%$, in very good agreement with the experimental results for the best overlap between the two fields. The simulations also show the presence of a peak that would result from a twophoton ATI absorption from the xuv field. Its relative magnitude is around $10^{-6}$ times that of the main peak. As expected, this two-photon peak is also accompanied by sidebands, which, interestingly, have relative intensities that decrease less markedly than those associated with the singlephoton-ionization signal.

The relative magnitude of the sidebands can also be calculated with reasonable accuracy through a simplified analysis based on the so-called soft-photon approximation. Within this framework, the differential cross section for a laserassisted process is obtained by multiplying the exact cross section for the process considered, in the absence of the dressing field, by $J_{n}^{2}\left(\vec{\alpha}_{0} \cdot \vec{k}\right)[21]$. Here $J_{n}(z)$ is a Bessel function, $\vec{k}$ is the wave vector of the photoelectron, $\vec{\alpha}_{0}=\vec{F}_{0} /\left(\omega^{2}\right)$ is the classical excursion vector of a free electron embedded within the dressing laser field $\vec{F}_{0} \sin (\omega t)$ of frequency $\omega$, and $n$ is the net number of photons exchanged with this dressing field (absorption $n>0$ or stimulated emission $n<0$ ). A value of $2.42 \%$ is obtained, to be compared to $1.95 \%$ derived from the TDSE treatment, for the relative magnitude of the righthand side sideband.

An interesting feature of the present two-color, twophoton experiment is that the sideband contains a combination of continua with $s$ and $d$ symmetries, which are not accessible by one-photon absorption from the ground state of He. Clearly, the detailed experimental study of two-photon processes, along with complementary theoretical investigations, provides deep insights into the dynamics of the atomic photoelectric effect. In particular, it allows extraction of the partial cross sections. In the present case, theory predicts for the high- (low-)kinetic-energy sideband a value 0.85 (2.0) for the ratio of the $\epsilon s$ to $\epsilon d$ partial photoionization cross sections. For an experimental verification of these values, measurements of the polarization dependence or of the angular distribution of the photoelectrons are needed (cf. [17,20]).

An important application of this class of two-color processes is that via a cross-correlation scheme it permits the determination of the relative delay between the xuv and the optical pulses on a subpicosecond time scale, which is an important aspect and necessary prerequisite for time-resolved experiments using the SASE FEL. The cross-correlation signal can be extracted from the data presented in Fig. 3, by integrating the overall intensity of the sideband and displaying this quantity as a function of the delay (Fig. 5). The data can be described by a Gaussian profile of full width at half maximum of $12.0 \pm 0.4 \mathrm{ps}$, which is, as expected, almost 


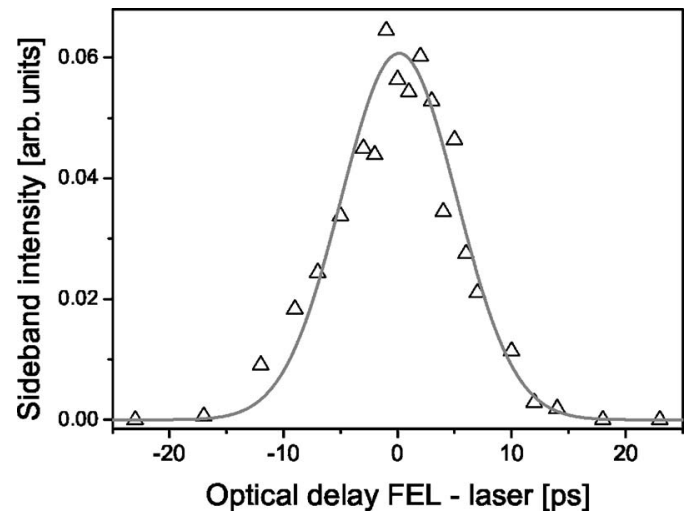

FIG. 5. Cross-correlation signal between the pulses of the FEL $(50 \mathrm{fs}, 32.2 \mathrm{~nm})$ and of the optical (12 ps, $523 \mathrm{~nm})$ laser.

completely related to the width of the optical laser. The convolution with the FEL pulses affects only slightly the overall resolution and the remaining discrepancy is probably due to the temporal instabilities of the FEL pulses with respect to those of the optical laser. The present data are in good agree- ment with a temporal jitter of the FEL of less than 1 ps as determined by the streak camera measurements.

The present two-color results illustrate the successful combination of the two very different laser types and clearly show the potential of short wavelength SASE FEL-optical laser pump-probe experiments. The results provide additional insight into two-color photon-atom interactions and demonstrate the single-shot capability of the experiment. Future pump-probe experiments of this type will be greatly facilitated through tuning of the wavelength of the FEL as well as the optical laser. In this way, we should be able to extend resonant photoionization and tuneable laser spectroscopy of the associated autoionization states to unprecedented interaction times and intensities.

We wish to thank the operator crew of the FEL for delivering the SASE beam. J.T.C. and P.O. acknowledge support from SFI and the HEA North South Programme, E.T.K. and P.Y. from Enterprise Ireland Grant No. SC/2003/180, and H.L. from the EU Marie Curie Programme. Support from the EU RTD project "X-Ray FEL Pump-Probe" Grant No. HRPI-CT-1999-50009 is gratefully acknowledged.
[1] J. Andruszkow et al., Phys. Rev. Lett. 85, 3825 (2000).

[2] H. Wabnitz et al., Nature (London) 420, 482 (2002).

[3] S. I. Themelis et al., J. Phys. B 37, 4281 (2004).

[4] P. Lambropoulos et al., Phys. Rev. A 72, 013410 (2005).

[5] V. Ayvazyan et al., Eur. Phys. J. D 37, 297 (2006).

[6] M. Drescher et al., Nature (London) 419, 803 (2002).

[7] L. B. Madsen et al., Phys. Rev. Lett. 85, 42 (2000).

[8] N. E. Karapanagioti et al., Phys. Rev. A 53, 2587 (1996).

[9] R. R. Jones and M. B. Campbell, Phys. Rev. A 61, 013403 (2000).

[10] H. Redlin et al. (unpublished).
[11] Ph. Wernet et al., Phys. Rev. A 64, 042707 (2001).

[12] D. Cubaynes et al., Phys. Rev. Lett. 92, 233002 (2004).

[13] J. M. Schins et al., Phys. Rev. Lett. 73, 2180 (1994).

[14] T. E. Glover et al., Phys. Rev. Lett. 76, 2468 (1996).

[15] R. Taïeb et al., Phys. Rev. A 62, 013402 (2000).

[16] E. S. Toma et al., Phys. Rev. A 62, 061801(R) (2000).

[17] P. O'Keeffe et al., Phys. Rev. A 69, 051401(R) (2004).

[18] S. Düsterer et al., Opt. Lett. 31, 1750 (2006).

[19] M. Gavrila et al., Phys. Rev. A 42, 236 (1990).

[20] O. Guyétand et al., J. Phys. B 38, 5313 (2005).

[21] N. M. Kroll and K. M. Watson, Phys. Rev. A 8, 804 (1973). 